\title{
Risk factors for disease progression in Japanese patients with COVID-19 with no or mild symptoms on admission
}

\author{
Toshifumi Ninomiya ${ }^{1,2 \dagger}{ }^{\dagger}$, Kohei Otsubo ${ }^{1 *}+$, Teppei Hoshino ${ }^{3}$, Mototsugu Shimokawa $^{4}$, Megumi Nakazawa ${ }^{2}$, \\ Yoriko Sato ${ }^{2}$, Hironori Mikumo ${ }^{2}$, Satoru Kawakami ${ }^{2,5}$, Shun Mizusaki ${ }^{1,2}$, Yusuke Mori $^{3}$, Hidenobu Arimura', \\ Yuko Tsuchiya-Kawano ${ }^{1}$, Koji Inoue ${ }^{1}$, Yujiro Uchida ${ }^{2}$ and Yoichi Nakanishi ${ }^{6}$
}

\begin{abstract}
Background: Although the risk factors for coronavirus disease 2019 (COVID-19) mortality have been identified, there is limited information about the risk factors for disease progression after hospitalization among Japanese patients with COVID-19 exhibiting no or mild symptoms.

Methods: All 302 consecutive patients who were admitted to our institutions and diagnosed with COVID-19 between March and December 2020 were retrospectively assessed. Ultimately, 210 adult patients exhibiting no or mild symptoms on admission were included in the analysis. They were categorized into the stable (no oxygen needed) and worsened (oxygen needed) groups, and their characteristics and laboratory data were compared.

Results: Among 210 patients, 49 progressed to a severe disease stage, whereas 161 did not. The mean patient age was 52.14 years, and 126 (60.0\%) patients were male. The mean body mass index (BMI) was $23.0 \mathrm{~kg} / \mathrm{m}^{2}$, and 71 patients were overweight $\left(\mathrm{BMI} \geq 25 \mathrm{~kg} / \mathrm{m}^{2}\right)$. Multivariate logistic analysis showed that old age, overweight, diabetes mellitus (DM), and high serum ferritin levels were independent risk factors for disease progression.
\end{abstract}

Conclusions: Clinicians should closely observe patients with COVID-19, especially those with risk factors such as old age, overweight, DM, and high serum ferritin levels, regardless of whether they have no or mild symptoms.

Keywords: COVID-19, Risk factors, Disease progression

\section{Background}

In December 2019, coronavirus disease 2019 (COVID$19)$, which is caused by severe acute respiratory syndrome coronavirus 2 (SARS-CoV-2), emerged in Wuhan, China, and subsequently spread globally, leading to a pandemic. In the early stage of the COVID-19 pandemic in Japan, most patients were hospitalized with or without

\footnotetext{
*Correspondence: kohei.otsubo@gmail.com

${ }^{\dagger}$ Toshifumi Ninomiya and Kohei Otsubo contributed equally to this study

1 Department of Respiratory Medicine, Kitakyushu Municipal Medical

Center, 2-1-1 Bashaku, Kokurakita-ku, Kitakyushu, Fukuoka 802-0077,

Japan

Full list of author information is available at the end of the article
}

symptoms. However, medical institutions were overwhelmed by the transmission of infection within a few months, and patients were recommended hospitalization, admission to a dedicated hotel, or home care on the basis of their symptoms and hospital availability at the discretion of the local health center and physicians. Although approximately $80 \%$ of COVID-19 cases are mild or asymptomatic, $20 \%$ develop pneumonia, and some progress to respiratory failure, acute respiratory distress syndrome, and multiple organ failure [1-4]. The reported risk factors for poor prognosis in COVID-19 include old age, male sex, obesity, comorbidities such as cardiovascular disease and diabetes mellitus (DM), and biomarkers, 
including C-reactive protein (CRP) level, lactate dehydrogenase (LDH) level, interleukin (IL)-6 level, ferritin level, D-dimer level, and neutrophil-to-lymphocyte ratio (NLR) [5-10]. However, little is known about the risk factors for developing severe disease after hospitalization among Japanese patients with COVID-19 exhibiting no or mild symptoms. Since March 2020, our two institutions of the Kitakyushu City Hospital Organization have accepted the largest number of patients with COVID-19 in Kitakyushu City. At our institution, patients without any symptoms or those with mild symptoms were only monitored or administered symptomatic treatment, such as antipyretics and antitussives, whereas oxygen supplementation was administered to patients who presented with low oxygen saturation on pulse oximetry $\left(\mathrm{SpO}_{2} \leq 93 \%\right.$ at rest). We have encountered some patients who developed respiratory failure and required oxygen administration or mechanical ventilation after hospitalization despite exhibiting no or mild symptoms on admission. Thus, this study aimed to retrospectively evaluate the risk factors for disease progression in patients with COVID-19 presenting with no or mild symptoms on admission.

\section{Methods}

\section{Study design and participants}

This multicenter, retrospective study was performed at Kitakyushu Municipal Medical Center and Kitakyushu City Yahata Hospital in Fukuoka, Japan. All 302 consecutive patients who were admitted to our institutions and diagnosed with COVID-19 between March 23, 2020, and December 31, 2020, were retrospectively assessed. Patients diagnosed with COVID-19 using polymerase chain reaction or antigen testing of nasopharyngeal swabs or sputum for SARS-CoV-2 and either did not exhibit any symptoms or had mild symptoms on admission were enrolled in this study. Pediatric patients $(<15$ years old $)$ and patients for whom data on specified physical characteristics (height, weight, and comorbidities) and laboratory tests (complete blood count, CRP, LDH, ferritin, and D-dimer) were unavailable were excluded. The follow-up period ended on the day when patients recovered and were discharged, were transferred to an advanced medical institution to receive intensive care, or died.

\section{Clinical analysis}

The following details were collected from the patient's medical records: age (per 10 years), sex, body mass index (BMI), comorbidities (asthma, hypertension, cardiovascular disease, DM, and interstitial lung disease), respiratory status, laboratory data (white blood cell count, neutrophil count, lymphocyte count, hemoglobin, CRP level, LDH level, ferritin level, and D-dimer level at admission), history of remdesivir and systemic corticosteroid usage before oxygen supplementation, and outcomes. All methods were performed in accordance with the relevant guidelines and regulations.

\section{Definition of disease status}

We classified the patients into the following two groups according to their respiratory status during the disease course: (1) worsened group, which comprised patients who needed oxygen administration because of pneumonia and low oxygen saturation on pulse oximetry $\left(\mathrm{SpO}_{2} \leq 93 \%\right.$ at rest) and (2) stable group, which comprised patients with no or mild symptoms who did not need oxygen administration.

\section{Statistical analysis}

The clinical characteristics of the patients were summarized using descriptive statistics and were compared using Fisher's exact test for categorical data and Student's $t$-test for continuous data. The risk factors for disease progression were evaluated using a logistic regression model. Multivariate logistic regression was performed using the backward elimination method. A P-value of $<0.05$ was considered statistically significant. Complete case analysis was adopted for all statistical analysis, and JMP Pro version 15 software for conducting statistical analysis (SAS Institute, Cary, NC, USA).

\section{Results}

\section{Patient characteristics and clinical course}

We excluded 14 pediatric patients, 47 patients who already required oxygen supplementation on admission, and 31 patients with incomplete data on specified physical characteristics and laboratory tests. Ultimately, 210 of the 302 patients were included in the analysis (Fig. 1). During hospitalization, 49 patients required oxygen supplementation (worsened group), whereas 161 patients remained stable until discharge (stable group). Among the 49 patients who exhibited disease progression, 4 died, but the remaining 45 recovered. The clinical characteristics of the patients on admission are shown in Table 1 . The mean age of the patients was 52.14 years (15-98 years), and $126(60.0 \%)$ of them were male. The mean BMI was $23.0 \mathrm{~kg} / \mathrm{m}^{2}$, and 71 patients were overweight $\left(\mathrm{BMI} \geq 25.0 \mathrm{~kg} / \mathrm{m}^{2}\right)$. The major comorbidities were hypertension (24.8\%), asthma (12.4\%), DM (11.0\%), and cardiovascular disease (11.0\%). None of the patients were administered remdesivir or systemic corticosteroids prior to oxygen supplementation.

\section{Risk factors for disease progression}

Table 2 summarizes the univariate and multivariate analyses results of the risk factors for disease progression. In univariate analysis, old age, male sex, $\mathrm{BMI} \geq 25 \mathrm{~kg} /$ $\mathrm{m}^{2}$, hypertension, cardiovascular disease, DM, CRP 


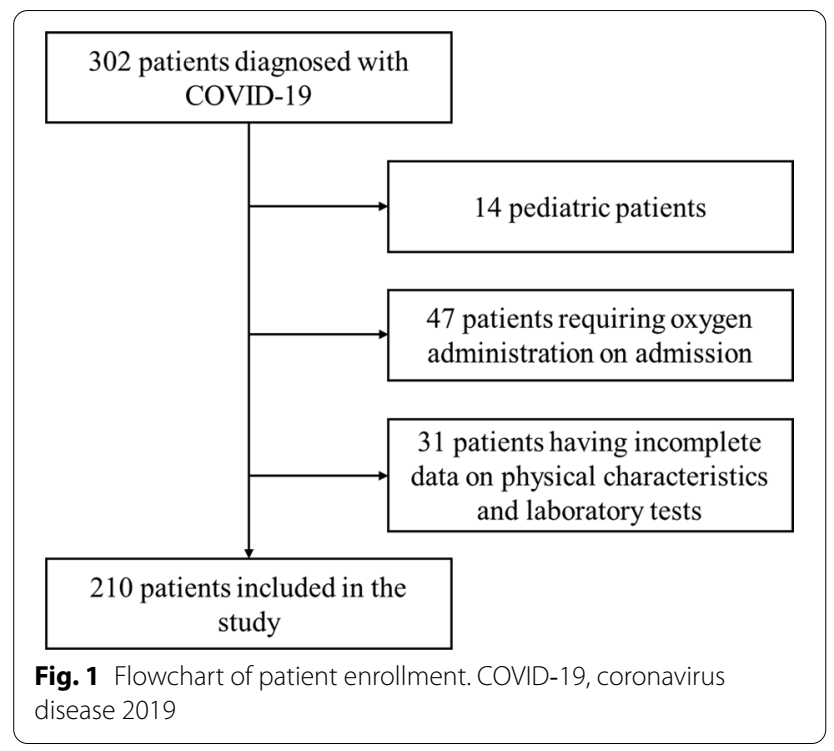

level, LDH level, creatinine level, and ferritin level were significantly associated with disease progression. In multivariate analysis, old age (odds ratio [OR] 1.538; 95\% confidence interval [CI] 1.219-1.949; $\mathrm{P}<0.001$ ), overweight (OR 3.260; 95\% CI 1.463-7.265; $\mathrm{P}=0.0038$ ), DM (OR 2.851; 95\% CI 1.056-7.698; $\mathrm{P}=0.039)$, and high serum ferritin levels (OR 1.002; 95\% CI 1.001-1.004; $\mathrm{P}<0.001)$ were found to be independent risk factors for disease progression.

\section{Discussion}

The risk factors for COVID-19 mortality have been extensively reported worldwide. Although reports on such risk factors in Japanese patients are limited, a nationwide COVID-19 inpatient registry, COVID-19 Registry Japan (COVIREGI-JP), was created in March 2020. Matsunaga et al. identified the clinical epidemiological characteristics of hospitalized patients with COVID-19 registered in COVIREGI-JP and reported that the mortality rate of these patients was $7.5 \%$, which was lower than that in other countries $(\sim 15-25 \%)[4,11,12]$. However, some Japanese patients with COVID-19 developed respiratory failure and required oxygen administration or mechanical ventilation despite having no or mild symptoms on admission. Hence, this study investigated the clinical characteristics of patients with COVID-19 with no or mild symptoms on admission. The results revealed that old age, overweight, DM, and high serum ferritin level

Table 1 Clinical characteristics of patients with COVID-19 on admission

\begin{tabular}{|c|c|c|c|c|}
\hline Characteristics & Total $(n=210)$ & Worsened $(n=49)$ & Stable $(n=161)$ & $P$ value \\
\hline Age, years & $52.14 \pm 20.14$ & $63.47 \pm 13.39$ & $48.69 \pm 20.58$ & $<0.001$ \\
\hline Sex, n (\%) & & & & 0.013 \\
\hline Male & $126(60.0)$ & $37(75.5)$ & $89(55.3)$ & \\
\hline Female & $84(40.0)$ & $12(24.5)$ & $72(44.7)$ & \\
\hline BMI, n (\%) & & & & $<0.001$ \\
\hline$<25 \mathrm{~kg} / \mathrm{m}^{2}$ & $139(66.2)$ & $20(40.8)$ & $119(73.9)$ & \\
\hline$\geq 25 \mathrm{~kg} / \mathrm{m}^{2}$ & $71(33.8)$ & $29(59.2)$ & $42(26.1)$ & \\
\hline \multicolumn{5}{|l|}{ Comorbidities, n (\%) } \\
\hline Hypertension & $52(24.8)$ & $25(51.0)$ & $27(16.8)$ & $<0.001$ \\
\hline Asthma & $26(12.4)$ & $6(12.2)$ & $20(12.4)$ & $>0.99$ \\
\hline Diabetes mellitus & $23(11.0)$ & $11(22.5)$ & $12(7.5)$ & 0.0071 \\
\hline Cardiovascular disease & $23(11.0)$ & $10(20.4)$ & $13(8.1)$ & 0.033 \\
\hline Interstitial lung disease & $5(2.4)$ & $0(0.0)$ & $5(3.1)$ & 0.59 \\
\hline \multicolumn{5}{|l|}{ Laboratory data } \\
\hline $\mathrm{WBC}, 10^{9} / \mathrm{L}$ & $5.19 \pm 2.07$ & $5.33 \pm 2.36$ & $5.15 \pm 1.94$ & 0.58 \\
\hline NLR & $4.21 \pm 8.26$ & $4.45 \pm 4.18$ & $4.32 \pm 5.35$ & 0.21 \\
\hline Hemoglobin, g/dL & $14.07 \pm 6.22$ & $13.88 \pm 1.93$ & $14.13 \pm 1.70$ & 0.37 \\
\hline $\mathrm{CRP}, \mathrm{mg} / \mathrm{dL}$ & $1.78 \pm 7.41$ & $3.55 \pm 3.89$ & $1.24 \pm 2.30$ & $<0.001$ \\
\hline $\mathrm{LDH}, \mathrm{U} / \mathrm{L}$ & $206.19 \pm 68.81$ & $245.35 \pm 3.89$ & $194.27 \pm 62.54$ & $<0.001$ \\
\hline Ferritin, ng/mL & $282.74 \pm 303.02$ & $507.24 \pm 437.22$ & $214.41 \pm 204.81$ & $<0.001$ \\
\hline D-dimer, $\mu \mathrm{g} / \mathrm{mL}$ & $1.31 \pm 7.87$ & $1.43 \pm 2.98$ & $1.28 \pm 4.14$ & 0.81 \\
\hline
\end{tabular}

Categorical data are presented as numbers (percentages) and were analyzed using Fisher's exact test. Continuous data are presented as mean \pm standard deviation and were analyzed using Student's $t$-test. $P<0.05$ was considered statistically significant

$B M I$ body mass index, $W B C$ white blood cell, NLR neutrophil-to-lymphocyte ratio, CRP C-reactive protein, $L D H$ lactate dehydrogenase 
Table 2 Risk factors for disease progression in patients with COVID-19

\begin{tabular}{llcc}
\hline Factor & \multicolumn{1}{l}{ Univariate } & Multivariate \\
\cline { 2 - 3 } & OR (95\% Cl) & $<0.001$ & OR (95\% Cl) \\
\hline Age (per 10 years) & $1.495(1.243-1.808)$ & 0.013 & $1.538(1.219-1.949)$ \\
Sex & $2.494(1.212-5.132)$ & $<0.001$ & \\
Overweight (BMI $\geq 25)$ & $4.108(2.103-8.025)$ & 0.97 & $3.260(1.463-7.265)$ \\
Asthma & $0.984(0.371-2.606)$ & $<0.001$ & \\
Hypertension & $5.170(2.577-10.37)$ & 0.019 & \\
Cardiovascular disease & $2.919(1.191-7.156)$ & 0.0050 & \\
Diabetes & $3.594(1.473-8.773)$ & 0.21 & \\
NLR & $1.057(0.970-1.153)$ & $<0.0001$ & \\
CRP & $1.272(1.132-1.430)$ & $<0.0001$ & \\
LDH & $1.010(1.005-1.015)$ & 0.0017 & \\
Creatinine & $9.551(2.327-39.20)$ & $<0.001$ & $1.002(1.001-1.004)$ \\
Ferritin & $1.003(1.002-1.004)$ & 0.81 & $<0.001$ \\
D-dimer & $1.009(0.936-1.088)$ &
\end{tabular}

Risk factors associated with disease severity were analyzed using a logistic regression model. $\mathrm{P}<0.05$ was considered statistically significant

$O R$ odds ratio, $C l$ confidence interval, $B M I$ body mass index, $N L R$ neutrophil-to-lymphocyte ratio, $C R P C$-reactive protein, $L D H$ lactate dehydrogenase

were risk factors for developing a severe form of COVID19 after hospitalization. Although these risk factors are mentioned in a previous COVIREGI-JP cohort study, our study was novel in that it was limited to patients without any symptoms or those having mild symptoms on admission and demonstrated that these are statistically significant risk factors for disease progression. Asymptomatic patients with COVID-19 or those with mild symptoms are often monitored at home or in a hotel rather than being hospitalized. Therefore, the clinical implication of our study is that whether these patients have the risk factors for disease progression identified in this study will help determine their monitoring facility (hospital, hotel, or home) at the time of diagnosis. Thereby, physicians can carefully follow-up on patients with risk factors for disease progression and provide immediate treatment on worsening of symptoms.

The OR for disease progression in patients who were overweight was 3.260 , which was the highest among statistically significant risk factors in this study. Obesity affects the respiratory function through different mechanisms, such as respiratory compromise, chronic inflammation, and complications. Obesity is also associated with pulmonary restriction, ventilation-perfusion mismatch, respiratory muscle fatigue, and sleep apnea syndrome; thus, patients with obesity have a background of hypoxemia and are more prone to respiratory failure [13]. These patients also have higher expression levels of tumor necrosis factor $\alpha$, IL-1, and IL-6. These proinflammatory cytokines increase the inflammatory response and abnormal $\mathrm{T}$ cell response, causing drastic lung injury and severe pneumonia [14]. When chronic hypoxia stimulates the hypoxia-inducible factor, detrimental effects occur on the immune system, as well as cytokine storm, which is associated with the poor outcomes of COVID-19 [15]. Overweight and obesity are risk factors of severe illness in patients with COVID-19 [16-18]. Therefore, one factor that can explain the low COVID19 mortality rate in Japan is that this country has a small obese population compared with Western countries. Our study also revealed that DM was a statistically significant risk factor for disease progression. Hyperglycemia causes immune dysfunction, including impaired neutrophil function, antioxidant system function, and humoral immunity; thus, patients with DM with COVID-19 have a high risk of disease progression [19, 20]. Furthermore, high serum ferritin levels at admission were significantly associated with the disease progression of COVID-19. Because serum ferritin is an iron-storage protein that regulates cellular oxygen metabolism, it is used as a marker for iron overload disorders, including hemochromatosis and hemosiderosis, which were not identified in patients enrolled in this study. It is also an immunomodulatory molecule with both immunosuppressive and proinflammatory functions that cause cytokine storms [21]. Elevated ferritin serum levels reportedly correlate with disease severity in patients with COVID-19 [22-24]. Therefore, patients with elevated serum ferritin levels are more likely to develop cytokine storms and subsequently, severe respiratory failure.

Recent studies have shown that high levels of D-dimer and NLR are risk factors for mortality in patients with COVID-19 [5, 10]. However, these factors were not significantly different in our study. Considering that 
these studies included patients who already had severe COVID-19 on admission, it is possible that high levels of D-dimer and NLR are the result of disease severity and are not risk factors for disease progression in patients without any symptoms or those with mild symptoms.

This study has some limitations. First, it is retrospective in nature and has a relatively small sample size. There were several cases with missing values, particularly in the early stage of the pandemic, and we excluded those cases and adopted complete case analysis. In multivariate analysis, we included all factors and applied backward elimination to maximize the statistical power. As the number of patients who experienced the defined outcomes was small and statistically underpowered, we cannot conclude that they are critical risk factors for disease progression. However, our findings are consistent with those of previous studies from other countries, and we believe that the factors found in this study are likely to be risk factors for disease progression in Japanese patients with COVID-19. A larger-scale study is required to confirm our results. Second, the effects of treatment given after admission were not considered. Owing to the lack of information about effective treatment in the early stages of the COVID-19 pandemic, various drugs, including inhaled ciclesonide, intravenous dexamethasone, remdesivir, and unapproved investigational drugs, have been tried. Thus, a prospective controlled trial is needed to assess the effectiveness of a particular treatment.

\section{Conclusions}

To the best of our knowledge, this is the first study in Japan to identify the risk factors for disease progression in patients with COVID-19 who exhibited no or mild symptoms on admission. Thus, clinicians should closely observe patients with COVID-19, especially those with risk factors such as old age, overweight, DM, and high serum ferritin levels, even if the patients are asymptomatic or exhibit mild symptoms.

\section{Abbreviations \\ COVID-19: Coronavirus disease 2019; BMI: Body mass index; SARS-CoV-2: Severe acute respiratory syndrome coronavirus 2; DM: Diabetes mellitus; CRP: C-reactive protein; LDH: Lactate dehydrogenase; NLR: Neutrophil-to- lymphocyte ratio.}

\section{Acknowledgements}

Not applicable.

\begin{abstract}
Authors' contributions
$\mathrm{TN}$ and $\mathrm{KO}$ were involved in study conception and design and were major contributors in writing the manuscript; $\mathrm{YU}$ and $\mathrm{YN}$ were involved in study conception and design; MS was involved in statistical evaluation of the study data; TN, KO, TH, MN, YS, HM, SK, SM, YM, HA, YT-K, KI, and YU were involved in data acquisition. All authors read and approved the final manuscript.
\end{abstract}

\section{Availability of data and materials}

The datasets used and/or analyzed during the current study are available from the corresponding author on reasonable request.

\section{Declarations}

Ethics approval and consent to participate

The Ethics Committee of Kitakyushu City Hospital Organization approved the study protocol. Given the retrospective design and the assurance of anonymity, the requirement of obtaining patient consent was waived by the Ethics Committee of Kitakyushu City Hospital Organization. Information about study participation was officially announced using posters. All patient data were anonymized prior to the analysis.

\section{Consent for publication}

Not applicable.

\section{Competing interests}

The authors declare that they have no competing interests.

\section{Author details}

'Department of Respiratory Medicine, Kitakyushu Municipal Medical Center, 2-1-1 Bashaku, Kokurakita-ku, Kitakyushu, Fukuoka 802-0077, Japan. ${ }^{2}$ Department of General Internal Medicine, Kitakyushu Municipal Medical Center, 2-1-1 Bashaku, Kokurakita-ku, Kitakyushu, Fukuoka 802-0077, Japan. ${ }^{3}$ Department of Internal Medicine, Kitakyushu City Yahata Hospital, 2-6-2, Ogura, Yahatahigashi-ku, Kitakyushu, Fukuoka 805-8534, Japan. ${ }^{4}$ Department of Biostatistics, Yamaguchi University Graduate School of Medicine, 1-1-1 Minamikogushi, Ube-shi, Yamaguchi 755-8505, Japan. ${ }^{5}$ Department of Respiratory Medicine, JCHO Kyushu Hospital, 1-8-1 Kishinoura, Yahatanishi-ku, Kitakyushu, Fukuoka 806-8501, Japan. ${ }^{6}$ Kitakyushu City Hospital Organization, 1-35 Furusenbamachi, Kokurakita-ku, Kitakyushu 802-0082, Japan.

Received: 19 May 2021 Accepted: 13 August 2021

Published online: 21 August 2021

\section{References}

1. Wiersinga WJ, Rhodes A, Cheng AC, Peacock SJ, Prescott HC. Pathophysiology, transmission, diagnosis, and treatment of coronavirus disease 2019 (COVID-19): a review. JAMA. 2020;324:782-93.

2. Wang C, Horby PW, Hayden FG, Gao GF. A novel coronavirus outbreak of global health concern. The Lancet. 2020;395:470-3.

3. Wu Z, McGoogan JM. Characteristics of and important lessons from the coronavirus disease 2019 (COVID-19) outbreak in China: summary of a report of 72314 cases from the Chinese center for disease control and prevention. JAMA. 2020;323:1239-42.

4. Huang C, Wang Y, Li X, et al. Clinical features of patients infected with 2019 novel coronavirus in Wuhan, China. The Lancet. 2020;395:497-506.

5. Du RH, Liang $L R$, Yang $C Q$, et al. Predictors of mortality for patients with COVID-19 pneumonia caused by SARS-CoV-2: a prospective cohort study. Eur Respir J. 2020;55:2000524.

6. Williamson EJ, Walker AJ, Bhaskaran K, et al. Factors associated with COVID-19-related death using OpenSAFELY. Nature. 2020;584:430-6.

7. Palaiodimos L, Kokkinidis DG, Li W, et al. Severe obesity, increasing age and male sex are independently associated with worse in-hospital outcomes, and higher in-hospital mortality, in a cohort of patients with COVID-19 in the Bronx, New York. Metabolism. 2020;108:154262.

8. Ayanian S, Reyes J, Lynn L, Teufel K. The association between biomarkers and clinical outcomes in novel coronavirus pneumonia in a US cohort. Biomark Med. 2020;14:1091-7.

9. Tian W, Jiang W, Yao J, et al. Predictors of mortality in hospitalized COVID-19 patients: a systematic review and meta-analysis. J Med Virol. 2020;92:1875-83

10. Liu Y, Du X, Chen J, et al. Neutrophil-to-lymphocyte ratio as an independent risk factor for mortality in hospitalized patients with COVID-19. J Infect. 2020;81:e6-12.

\section{Funding}

No funding was received for this study. 
11. Docherty AB, Harrison EM, Green CA, et al. Features of 20133 UK patients in hospital with covid-19 using the ISARIC WHO Clinical Characterisation Protocol: prospective observational cohort study. BMJ. 2020;369:m1985.

12. Richardson S, Hirsch JS, Narasimhan M, et al. Presenting characteristics, comorbidities, and outcomes among 5700 patients hospitalized with COVID-19 in the New York City Area. JAMA. 2020;323:2052-9.

13. Kwok S, Adam S, Ho JH, et al. Obesity: a critical risk factor in the COVID-19 pandemic. Clin Obes. 2020;10:e12403.

14. Chiappetta S, Sharma AM, Bottino V, Stier C. COVID-19 and the role of chronic inflammation in patients with obesity. Int J Obes (Lond). 2020;44:1790-2.

15. AbdelMassih A, Yacoub E, Husseiny RJ, et al. Hypoxia-inducible factor (HIF): the link between obesity and COVID-19. Obes Med. 2021;22:100317.

16. Suresh S, Siddiqui M, Abu Ghanimeh M, et al. Association of obesity with illness severity in hospitalized patients with COVID-19: a retrospective cohort study. Obes Res Clin Pract. 2021;15:172-6.

17. Kalligeros M, Shehadeh F, Mylona EK, et al. Association of obesity with disease severity among patients with coronavirus disease 2019. Obesity (Silver Spring). 2020;28:1200-4.

18. Wang J, Zhu L, Liu L, et al. Overweight and obesity are risk factors of severe illness in patients with COVID-19. Obesity (Silver Spring) 2020;28:2049-55.
19. Geerlings SE, Hoepelman Al. Immune dysfunction in patients with diabetes mellitus (DM). FEMS Immunol Med Microbiol. 1999;26:259-65.

20. Casqueiro J, Casqueiro J, Alves C. Infections in patients with diabetes mellitus: a review of pathogenesis. Indian J Endocrinol Metab. 2012;16(Suppl 1):S27-36.

21. Kernan KF, Carcillo JA. Hyperferritinemia and inflammation. Int Immunol. 2017;29:401-9.

22. Qeadan F, Tingey B, Gu LY, Packard AH, Erdei E, Saeed Al. Prognostic values of serum ferritin and D-dimer trajectory in patients with COVID-19. Viruses. 2021;13:419.

23. Colafrancesco S, Alessandri C, Conti F, Priori R. COVID-19 gone bad: a new character in the spectrum of the hyperferritinemic syndrome? Autoimmun Rev. 2020;19:102573.

24. Taneri PE, Gomez-Ochoa SA, Llanaj E, et al. Anemia and iron metabolism in COVID-19: a systematic review and meta-analysis. Eur J Epidemiol. 2020;35:763-73.

\section{Publisher's Note}

Springer Nature remains neutral with regard to jurisdictional claims in published maps and institutional affiliations.
Ready to submit your research? Choose BMC and benefit from:

- fast, convenient online submission

- thorough peer review by experienced researchers in your field

- rapid publication on acceptance

- support for research data, including large and complex data types

- gold Open Access which fosters wider collaboration and increased citations

- maximum visibility for your research: over $100 \mathrm{M}$ website views per year

At BMC, research is always in progress.

Learn more biomedcentral.com/submissions 